\title{
Standardization of Packaging Material and Storage Condition for Pomegranate Leather
}

\author{
Kaustubh Das, Mohit Kumar and Ankan Das* \\ Department of Horticulture, Institute of Agricultural Science, University of Calcutta, 51/2 \\ Hazra Road, Kolkata-700019, India \\ *Corresponding author
}

\section{Keywords}

Leather, packaging, refrigerated, ambient, study

Article Info

Accepted:

22 July 2019

Available Online:

10 August 2019

\section{A B S T R A C T}

Pomegranate fruits were used to prepare pomegranate fruit leather. Thereafter packaging of the pomegranate leather was carried in variable materials or combinations. Finally storage of the sealed packets containing the leather was carried in refrigerated and ambient conditions. Laboratorial analysis from the fruit leather stored in both the situations was carried out at recurrent breaks throughout the experiment. From the study it was observed that among the storage situation the refrigerated condition was better over the other. Regarding the packaging material, it was found that packaging material with combination of Styrofoam and Low Density Poly Ethylene and another packaging material, plastic punnet box were very much fit for the long keeping of the pomegranate leather for both the storage environment.

\section{Introduction}

Pomegranate is one such fruit which is widely rich in various nutrients. However the anatomy of the fruit like its outside thick rind which encloses the juicy arils inside, sometimes makes it difficult to consume. As the arils are the edible part of the fruit, extraction of it becomes very hectic and time taking in a situation where everyone today is very much occupied. Furthermore the high moisture content of the arils imposes a challenge in their long keeping. Dehydration thus can become a very important technology by which this exorbitant moisture content can be brought down to safe ranges, which will automatically result in lowering the microbial contamination and in turn the product can be kept throughout a long storage time (Kordylas, 1990). By using dehydration technology, different types of final commodities or products can be developed. Fruit leather is one such thing which can be obtained as an output of dehydration. But alone dehydration is not enough to preserve the product. It requires efficient packaging by suitable materials to enhance the storage or shelf life. Proper packaging also helps in providing the internal 
contents, adequate protection from different undesirable conditions which may arise during movement of the commodities and trading (Wills et al., 1989; Irtwenge, 2006). After packaging the optimum storage condition or temperature is also important which also plays an indispensible role in the products longevity.

Thus considering these points, in the present study pomegranate leather was prepared followed by packaging with different packaging materials and thereafter storage was done at ambient as well as refrigerated condition. The main objective of the experiment was to standardize an adequate packaging material and optimum storage condition for the prepared dehydrated product.

\section{Materials and Methods}

The experiment with respect to the mentioned title was carried out in the Department of Horticulture, Institute of Agricultural Science, University of Calcutta, Kolkata, West Bengal during the academic year of 2018-19. The mature pomegranate fruits were brought to the laboratory of the Department. Then after washing the fruits, arils from them were manually brought out and crushed in a mixture grinder, until a fine paste/mixture was obtained.

Then the mixture was boiled and 20 grams pectin powder was added per liter of the pomegranate paste. After that the mixture was allowed to cool with addition of $1 \%$ ascorbic acid and then spread over aluminum trays (wrapped internally with aluminium foil) and placed inside a hot air oven for dehydration at $60^{\circ} \mathrm{C}$.

The materials took 14.5 hours for complete dehydration. Then the prepared leathers were packed inside packets prepared from the following packaging materials/combinations used as treatments in the study.

\section{Number of treatments}

$\mathrm{T}_{1^{-}}$HDPE (High Density Poly Ethylene), $\mathrm{T}_{2^{-}}$ Styrofoam, $\mathrm{T}_{3^{-}}$Brown paper, $\mathrm{T}_{4^{-}}$Plastic punnet box, $\mathrm{T}_{5}-$ Styrofoam + LDPE (Low Density Poly Ethylene), $\mathrm{T}_{6}$ - LDPE + HDPE.

After that, the storage of the pomegranate leathers (packed and sealed in different packaging treatments) was carried out in ambient (temperature $27^{ \pm} 2{ }^{0} \mathrm{C}$ ) as well as in refrigerated conditions (temperature: $8+2{ }^{0} \mathrm{C}$ ).

\section{Experimental design}

All the treatments were replicated three times and 2 factorial CRD was used as the design of experiment. Help of an online statistical software was taken for the study (Sheoran et al., 1998).

\section{Observations}

Different parameters were analyzed from all the pomegranate leathers of refrigerated as well as of ambient storage conditions at the intervals of $0,15,30,45 \& 60$ days. The attributes which were taken into account for the study were total soluble solids (Ranganna, 2003), reducing sugar (Ranganna, 2003), total sugar (Ranganna, 2003), titratable acidity (Ranganna, 2003), radical scavenging activity (Brand-williams et al., 1995), total phenolic content (Singleton et al., 1999) and total fungal count (Allen, 1953).

\section{Results and Discussion}

Total Soluble Solids for the pomegranate leathers (Table 1) packaged in different packaging materials under refrigerated storage condition, at 0 days was observed at $6^{0}$ Brix. By the end of the storage the TSS increased to a maximum of $7^{0}$ Brix for pomegranate leather packed in treatments $\mathrm{T}_{1}$ (HDPE), $\mathrm{T}_{2}$ (Styrofoam), $\mathrm{T}_{3}$ (Brown paper) and $\mathrm{T}_{6}$ (LDPE 
+ HDPE). For pomegranate leathers packaged with $\mathrm{T}_{4}$ (Plastic punnet box) and $\mathrm{T}_{5}$ (Styrofoam + LDPE), the TSS content was observed at $6.67^{0}$ Brix (at 60 days after storage). For the pomegranate leathers stored at ambient condition the TSS range at 15 days of storage was very much similar to the values obtained at 15 days of storage of pomegranate leathers at refrigerated condition. The increasing pattern was seen for other storage intervals and at 60 days of storage, leather packed in $\mathrm{T}_{4}$ (Plastic punnet box) resulted with lowest value of $7.67^{\circ}$ Brix and content inside $\mathrm{T}_{3}$ (Brown paper) was observed with TSS value of $9.67^{0}$ Brix respectively. Reducing sugar and total sugar percentage (Table 2 and 3) increased in all the treatments for both the storage conditions during the experiment. However, the accumulation of reducing and total sugar content was less for the pomegranate leather inside packaging treatments stored at refrigerated condition, in comparison to ambient storage where higher rates of sugar gain was seen for the packed dehydrated product. Among the treatments it was observed that pomegranate leathers by packaging with $\mathrm{T}_{5}$ (Styrofoam + LDPE) showed lower buildup of sugar levels for the both the storage condition throughout the study analysis interval of 15, 30, 45 and 60 days of analysis. Whereas pomegranate leather packaged with treatment $T_{3}$ (Brown paper) showed the higher reducing and total sugar uptake at different day's interval of storage analysis for both the conditions.

The titratable acid content (Table 4) increased for the fruit leather during the storage analysis for both the storage conditions. For the pomegranate leather stored at refrigerated condition, the acid gain was there, but the accumulation was very marginal amongst the different packaging treatments (containing the fruit leather) at different days of analysis. At 15 day interval pomegranate leather with $T_{5}$ (Styrofoam + LDPE) and $\mathrm{T}_{4}$ (Plastic punnet box) showed the lowest acid values of $0.06 \%$ and identical values of $0.12 \%$ was observed for other packaging treatments. The acid content from this point increased slightly for the dehydrated product with different treatments (refrigerated condition) and finally at 60 days of storage, pomegranate leather packed with $\mathrm{T}_{5}$ (Styrofoam + LDPE) recorded the lowest values of $0.19 \%$. For the packed pomegranate leather which was stored at ambient condition, the gain in the content of titratable acidity was in a bit a higher side. Here by 60 days of storage, pomegranate leather packed with $\mathrm{T}_{4}$ (Plastic punnet box) gave the least accumulation of $0.30 \%$ which was followed by pomegranate leather packed with $\mathrm{T}_{5}$ (Styrofoam + LDPE), showing $0.32 \%$ of acid content. Pomegranate leather packed with $\mathrm{T}_{1}$ (HDPE), $\mathrm{T}_{3}$ (Brown paper) and $\mathrm{T}_{6}$ (LDPE + HDPE) recorded the highest acid content of $0.42 \%$.

The antioxidant (percentage inhibition of DPPH) content declined during the storage intervals for the pomegranate leathers packed with different treatments under both the storage conditions (Table 5). For the pomegranate leather stored at refrigerated condition, antioxidant level (percentage inhibition of DPPH) at 15 day of storage where more or less similar for all the treatments, however a gradual decline was observed thereafter. At the end of experiment it was observed that (for refrigerated storage) leathers which were packed in packaging treatments $\mathrm{T}_{5}$ (Styrofoam + LDPE) and $\mathrm{T}_{4}$ (Plastic punnet box) recorded with maximum value of 74.23 and pomegranate leather packed in treatments $T_{1}$ (HDPE), $T_{3}$ (Brown paper), $\mathrm{T}_{6}$ (LDPE + HDPE) showed the least amount of 66.83. For pomegranate leathers packed with different materials with storage at ambient condition, again declination with respect to antioxidant (percentage inhibition of DPPH) level throughout the storage period of analysis was recorded. 
Fig.1 ( $T_{1}-$ HDPE, $T_{2}-$ Styrofoam, $T_{3}$ - Brown paper, $T_{4}$ - Plastic punnet box, $T_{5}-$ Styrofoam + LDPE, T6- LDPE + HDPE)

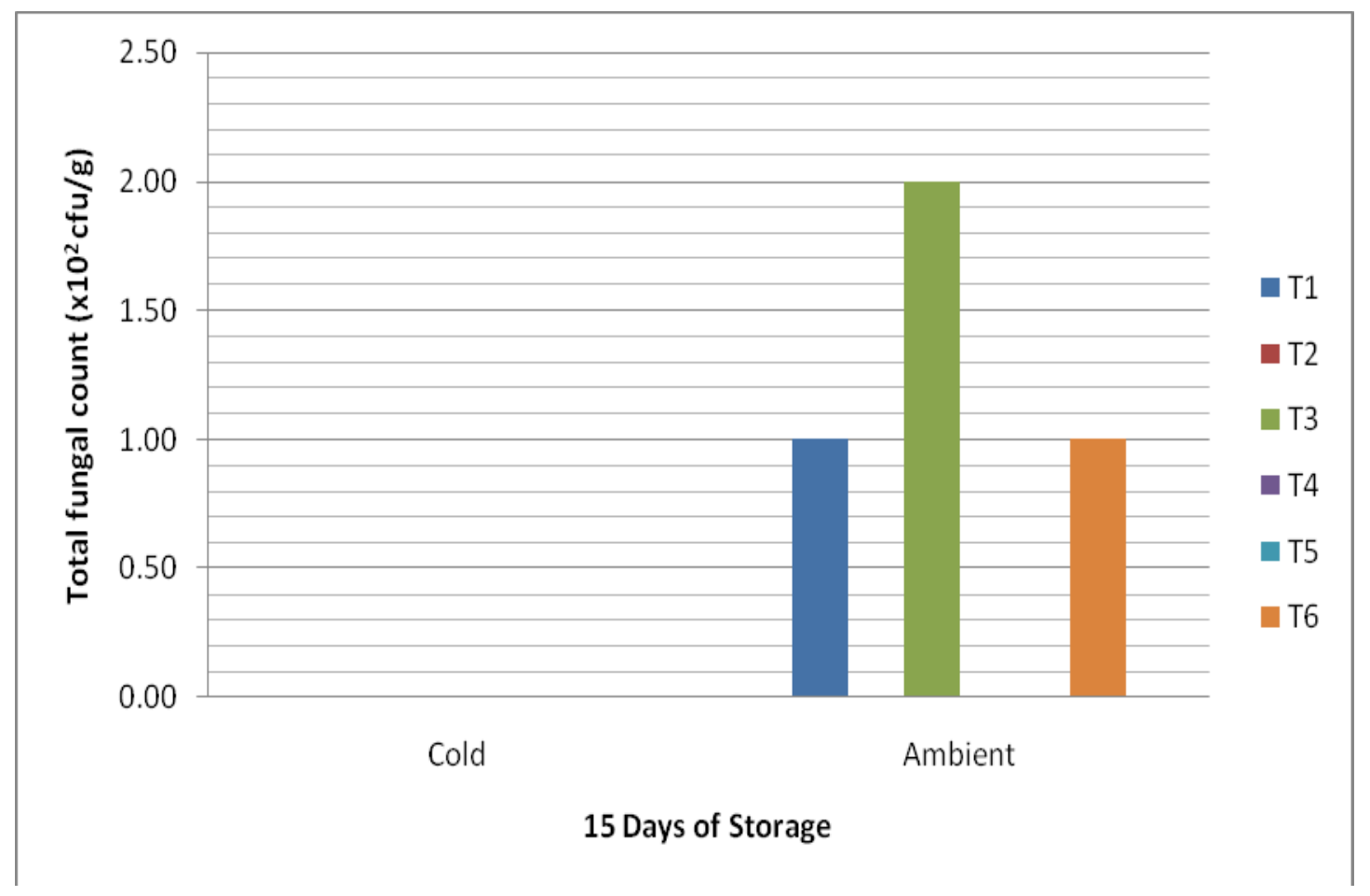

Fig.2 ( $T_{1}$ - HDPE, $T_{2}$-Styrofoam, $\mathrm{T}_{3}$ - Brown paper, $\mathrm{T}_{4}$ - Plastic punnet box, $\mathrm{T}_{5^{-}}$Styrofoam + LDPE, $\left.\mathrm{T}_{6}-\mathrm{LDPE}+\mathrm{HDPE}\right)$

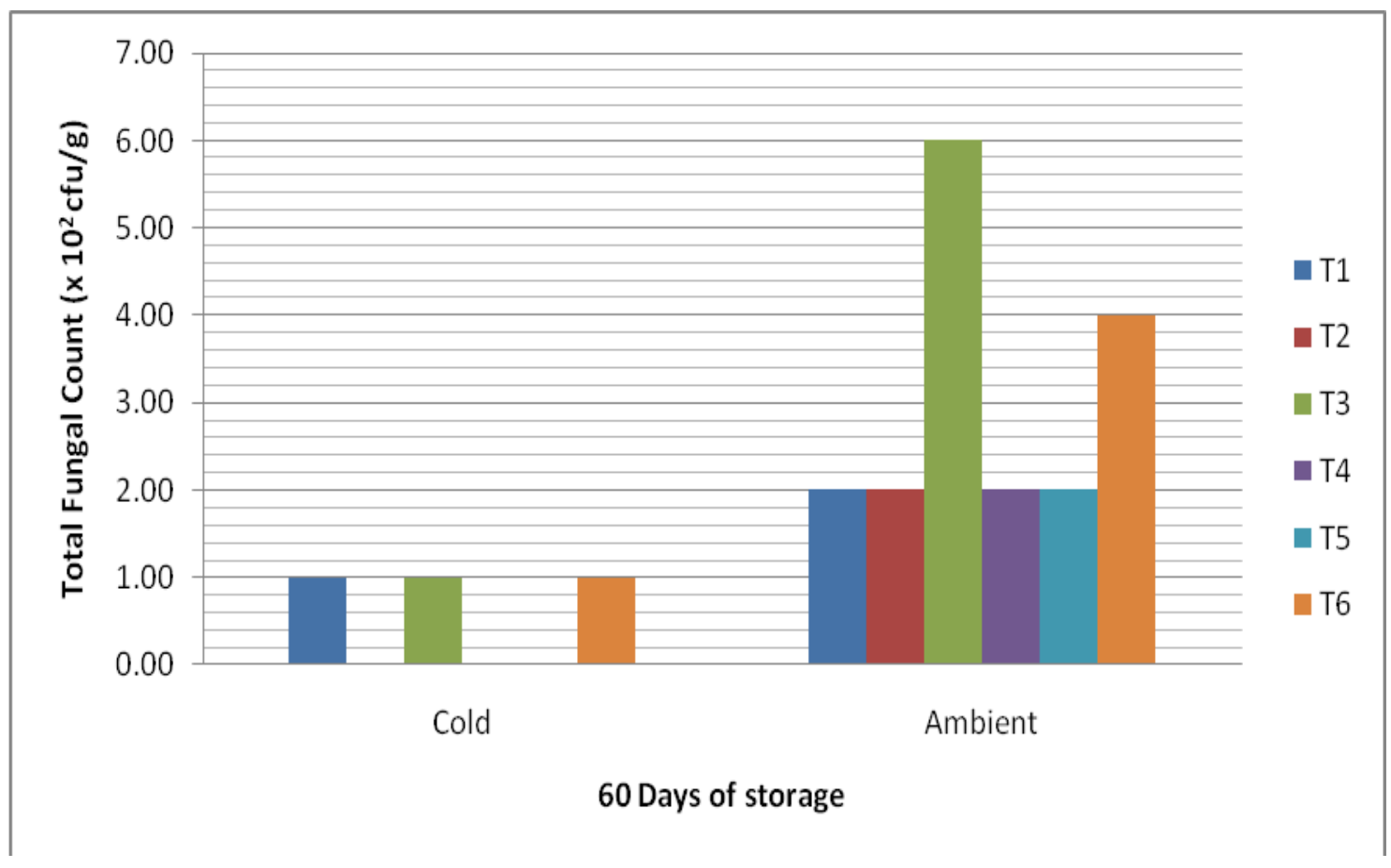


Table.1 TSS ( ${ }^{0}$ Brix) of pomegranate leathers packaged in different packaging treatments in cold and ambient storage condition at periodic intervals during storage

\begin{tabular}{|c|c|c|c|c|c|c|c|c|c|c|c|c|c|c|c|c|}
\hline 0 DAS & 15 DAS & Cold & $\begin{array}{c}\text { Ambi } \\
\text { ent }\end{array}$ & $\begin{array}{c}\text { Mea } \\
\text { n A }\end{array}$ & 30 DAS & Cold & $\begin{array}{c}\text { Amb } \\
\text { ient }\end{array}$ & $\begin{array}{c}\text { Mean } \\
\text { A }\end{array}$ & $\begin{array}{c}45 \\
\text { DAS }\end{array}$ & $\begin{array}{c}\text { Col } \\
\text { d }\end{array}$ & $\begin{array}{c}\text { Amb } \\
\text { ient }\end{array}$ & $\begin{array}{c}\text { Mea } \\
\text { n A }\end{array}$ & 60 DAS & Cold & $\begin{array}{c}\text { Amb } \\
\text { ient }\end{array}$ & $\begin{array}{c}\text { Mean } \\
\text { A }\end{array}$ \\
\hline \multirow{11}{*}{$\begin{array}{c}A(1-6) \\
\times B(1- \\
2)=6.00\end{array}$} & $\mathbf{T}_{1}$ & 6.33 & 6.67 & 6.50 & $\mathbf{T}_{1}$ & 6.33 & 7.33 & 6.83 & $\mathbf{T}_{1}$ & 7.00 & 8.00 & 7.50 & $\mathbf{T}_{1}$ & 7.00 & 9.00 & 8.00 \\
\hline & $\mathbf{T}_{2}$ & 6.00 & 6.00 & 6.00 & $\mathbf{T}_{2}$ & 6.33 & 7.00 & 6.67 & $\mathbf{T}_{2}$ & 6.33 & 7.67 & 7.00 & $\mathbf{T}_{2}$ & 7.00 & 8.67 & 7.83 \\
\hline & $\mathbf{T}_{3}$ & 6.67 & 6.67 & 6.67 & $\mathbf{T}_{3}$ & 6.67 & 7.67 & 7.17 & $\mathbf{T}_{3}$ & 7.00 & 8.00 & 7.50 & $\mathbf{T}_{3}$ & 7.00 & 9.67 & 8.33 \\
\hline & $\mathbf{T}_{4}$ & 6.00 & 6.00 & 6.00 & $\mathbf{T}_{4}$ & 6.00 & 7.00 & 6.50 & $\mathbf{T}_{4}$ & 6.33 & 7.33 & 6.83 & $\mathbf{T}_{4}$ & 6.67 & 7.67 & 7.17 \\
\hline & $\mathbf{T}_{5}$ & 6.00 & 6.00 & 6.00 & $\mathbf{T}_{5}$ & 6.00 & 6.67 & 6.33 & $\mathbf{T}_{5}$ & 6.00 & 7.33 & 6.67 & $\mathbf{T}_{5}$ & 6.67 & 8.00 & 7.33 \\
\hline & $\mathbf{T}_{6}$ & 6.33 & 6.33 & 6.33 & $\mathbf{T}_{6}$ & 6.33 & 7.00 & 6.67 & $\mathbf{T}_{6}$ & 6.67 & 8.00 & 7.33 & $\mathbf{T}_{6}$ & 7.00 & 9.00 & 8.00 \\
\hline & $\begin{array}{c}\text { Mean } \\
\text { B }\end{array}$ & 6.22 & 6.28 & & Mean B & 6.28 & 7.11 & & $\begin{array}{c}\text { Mean } \\
\text { B }\end{array}$ & 6.56 & 7.72 & & Mean B & 6.89 & 8.67 & \\
\hline & Factors & C.D. & $\operatorname{SE}(d)$ & $\begin{array}{l}\text { SE } \\
(\mathbf{m})\end{array}$ & Factors & C.D. & $\begin{array}{c}\text { SE }(d \\
)\end{array}$ & $\mathrm{SE}(\mathrm{m})$ & $\begin{array}{c}\text { Factor } \\
\mathrm{s}\end{array}$ & C.D. & $\begin{array}{l}\text { SE } \\
\text { (d) }\end{array}$ & $\begin{array}{l}\text { SE } \\
(\mathrm{m})\end{array}$ & Factors & C.D. & $\begin{array}{l}\text { SE } \\
\text { (d) }\end{array}$ & $\mathrm{SE}(\mathrm{m})$ \\
\hline & $\begin{array}{c}\text { Factor } \\
\text { (A) }\end{array}$ & 0.489 & 0.236 & 0.167 & $\begin{array}{c}\text { Factor } \\
\text { (A) }\end{array}$ & N/A & 0.255 & 0.18 & $\begin{array}{c}\text { Factor } \\
\text { (A) }\end{array}$ & $\begin{array}{c}0.48 \\
9\end{array}$ & 0.236 & $\begin{array}{c}0.16 \\
7\end{array}$ & $\begin{array}{c}\text { Factor } \\
\text { (A) }\end{array}$ & 0.447 & 0.215 & 0.152 \\
\hline & $\begin{array}{c}\text { Factor } \\
\text { (B) }\end{array}$ & N/A & 0.136 & 0.096 & $\begin{array}{c}\text { Factor } \\
\text { (B) }\end{array}$ & 0.305 & 0.147 & 0.104 & $\begin{array}{c}\text { Factor } \\
\text { (B) }\end{array}$ & $\begin{array}{c}0.28 \\
3\end{array}$ & 0.136 & $\begin{array}{c}0.09 \\
6\end{array}$ & $\begin{array}{c}\text { Factor } \\
\text { (B) }\end{array}$ & 0.258 & 0.124 & 0.088 \\
\hline & $\begin{array}{l}\text { Factor( } \\
\text { A X B) }\end{array}$ & N/A & 0.133 & 0.236 & $\begin{array}{c}\text { Factor(A } \\
\text { X B) }\end{array}$ & N/A & 0.36 & 0.255 & $\begin{array}{c}\text { Factor } \\
(\text { A X } \\
\text { B) }\end{array}$ & N/A & 0.333 & $\begin{array}{c}0.23 \\
6\end{array}$ & $\begin{array}{l}\text { Factor( } \\
\text { A X B) }\end{array}$ & 0.632 & 0.304 & 0.215 \\
\hline
\end{tabular}

( $\mathrm{T}_{1}$ - HDPE, $\mathrm{T}_{2}$-Styrofoam, $\mathrm{T}_{3}$ - Brown paper, $\mathrm{T}_{4}$ - Plastic punnet box, $\mathrm{T}_{5^{-}}$Styrofoam + LDPE, $\mathrm{T}_{6}$ - LDPE + HDPE) 
Table.2 Reducing sugar (\%) of pomegranate leathers packaged in different packaging treatments in cold and ambient storage condition at periodic intervals during storage

\begin{tabular}{|c|c|c|c|c|c|c|c|c|c|c|c|c|c|c|c|c|}
\hline 0 DAS & 15 DAS & Cold & $\begin{array}{c}\text { Amb } \\
\text { ient }\end{array}$ & $\begin{array}{c}\text { Mean } \\
\text { A }\end{array}$ & 30 DAS & $\begin{array}{c}\text { Col } \\
\text { d }\end{array}$ & $\begin{array}{c}\text { Ambie } \\
\text { nt }\end{array}$ & $\begin{array}{c}\text { Mean } \\
\text { A }\end{array}$ & 45 DAS & Cold & $\begin{array}{c}\text { Am } \\
\text { bien } \\
\mathbf{t}\end{array}$ & $\begin{array}{c}\text { Mean } \\
\text { A }\end{array}$ & 60 DAS & $\begin{array}{c}\text { Col } \\
\text { d }\end{array}$ & $\underset{\text { nt }}{\text { Ambie }}$ & $\begin{array}{c}\text { Mean } \\
\text { A }\end{array}$ \\
\hline \multirow{11}{*}{$\begin{array}{l}A(1-6) \\
x \text { B (1- } \\
2)= \\
8.05\end{array}$} & $\mathbf{T}_{1}$ & 10.06 & 10.28 & 10.17 & $\mathbf{T}_{1}$ & $\begin{array}{c}10.2 \\
8\end{array}$ & 12.50 & 11.39 & $\mathbf{T}_{1}$ & 10.73 & $\begin{array}{c}12.6 \\
3\end{array}$ & 11.68 & $\mathbf{T}_{1}$ & $\begin{array}{c}12.6 \\
3\end{array}$ & 14.48 & 13.56 \\
\hline & $\mathbf{T}_{2}$ & 8.12 & 10.06 & 9.09 & $\mathbf{T}_{2}$ & $\begin{array}{c}10.2 \\
8\end{array}$ & 10.28 & 10.28 & $\mathbf{T}_{2}$ & 10.28 & $\begin{array}{c}12.5 \\
0\end{array}$ & 11.39 & $\mathbf{T}_{2}$ & $\begin{array}{c}12.5 \\
0\end{array}$ & 13.69 & 13.09 \\
\hline & $\mathbf{T}_{\mathbf{3}}$ & 10.06 & 10.28 & 10.17 & $\mathbf{T}_{\mathbf{3}}$ & $\begin{array}{c}10.2 \\
8\end{array}$ & 12.50 & 11.39 & $\mathbf{T}_{\mathbf{3}}$ & 11.50 & $\begin{array}{c}12.6 \\
3\end{array}$ & 12.07 & $\mathbf{T}_{3}$ & $\begin{array}{c}12.6 \\
3\end{array}$ & 14.48 & 13.56 \\
\hline & $\mathbf{T}_{4}$ & 8.12 & 10.06 & 9.09 & $\mathbf{T}_{4}$ & 8.12 & 10.28 & 9.20 & $\mathbf{T}_{4}$ & 10.28 & $\begin{array}{c}12.5 \\
0\end{array}$ & 11.39 & $\mathbf{T}_{4}$ & $\begin{array}{c}12.5 \\
0\end{array}$ & 13.69 & 13.09 \\
\hline & $\mathbf{T}_{5}$ & 8.12 & 10.06 & 9.09 & $\mathbf{T}_{5}$ & 8.12 & 10.28 & 9.20 & $\mathbf{T}_{5}$ & 10.06 & $\begin{array}{c}11.2 \\
4\end{array}$ & 10.65 & $\mathbf{T}_{5}$ & $\begin{array}{c}10.2 \\
8\end{array}$ & 12.50 & 11.39 \\
\hline & $\mathbf{T}_{6}$ & 10.06 & 10.06 & 10.06 & $\mathbf{T}_{6}$ & $\begin{array}{c}10.2 \\
8\end{array}$ & 12.50 & 11.39 & $\mathbf{T}_{6}$ & 10.73 & $\begin{array}{c}12.6 \\
3\end{array}$ & 11.68 & $\mathbf{T}_{6}$ & $\begin{array}{c}12.5 \\
0\end{array}$ & 13.69 & 13.09 \\
\hline & Mean B & 9.09 & 10.13 & & Mean B & 9.56 & 11.39 & & $\begin{array}{c}\text { Mean } \\
\text { B }\end{array}$ & 10.60 & $\begin{array}{c}12.3 \\
6\end{array}$ & & Mean B & $\begin{array}{c}12.1 \\
7\end{array}$ & 13.75 & \\
\hline & Factors & C.D. & $\begin{array}{l}\text { SE } \\
\text { (d) }\end{array}$ & $\begin{array}{l}\text { SE } \\
(\mathbf{m})\end{array}$ & Factors & C.D. & SE (d) & $\begin{array}{l}\text { SE } \\
(\mathbf{m})\end{array}$ & Factors & C.D. & $\begin{array}{l}\mathrm{SE}( \\
\text { d) }\end{array}$ & $\begin{array}{l}\text { SE } \\
(\mathbf{m})\end{array}$ & Factors & C.D. & $\mathrm{SE}(\mathrm{d})$ & $\mathrm{SE}(\mathrm{m})$ \\
\hline & $\begin{array}{l}\text { Factor } \\
\text { (A) }\end{array}$ & 0.197 & 0.095 & 0.067 & $\begin{array}{l}\text { Factor } \\
\text { (A) }\end{array}$ & 1.93 & 0.93 & 0.657 & $\begin{array}{l}\text { Factor } \\
\text { (A) }\end{array}$ & 0.104 & $\begin{array}{c}0.05 \\
0\end{array}$ & 0.035 & $\begin{array}{l}\text { Factor } \\
\text { (A) }\end{array}$ & $\begin{array}{c}0.11 \\
0\end{array}$ & 0.053 & 0.037 \\
\hline & $\begin{array}{l}\text { Factor( } \\
\text { B) }\end{array}$ & 0.114 & 0.055 & 0.039 & $\begin{array}{l}\text { Factor( } \\
\text { B) }\end{array}$ & $\begin{array}{c}1.11 \\
4\end{array}$ & 0.537 & 0.38 & $\begin{array}{l}\text { Factor } \\
\text { (B) }\end{array}$ & 0.060 & $\begin{array}{c}0.02 \\
9\end{array}$ & 0.020 & $\begin{array}{c}\text { Factor } \\
\text { (B) }\end{array}$ & $\begin{array}{c}0.06 \\
4\end{array}$ & 0.031 & 0.022 \\
\hline & $\begin{array}{c}\text { Factor( } \\
\text { A X B) }\end{array}$ & 0.278 & 0.134 & 0.095 & $\begin{array}{l}\text { Factor } \\
\text { (A X B) }\end{array}$ & N/A & 1.315 & 0.93 & $\begin{array}{c}\text { Factor } \\
\text { (A X B) }\end{array}$ & 0.147 & $\begin{array}{c}0.07 \\
1\end{array}$ & 0.050 & $\begin{array}{c}\text { Factor(A } \\
\text { X B) }\end{array}$ & $\begin{array}{c}0.15 \\
6\end{array}$ & 0.075 & 0.053 \\
\hline
\end{tabular}

( $\mathrm{T}_{1}$ - HDPE, $\mathrm{T}_{2}$-Styrofoam, $\mathrm{T}_{3}$ - Brown paper, $\mathrm{T}_{4}$ - Plastic punnet box, $\mathrm{T}_{5}$ - Styrofoam + LDPE, $\mathrm{T}_{6}$ - LDPE + HDPE) 
Table.3 Total sugar (\%) of pomegranate leathers packaged in different packaging treatments in cold and ambient storage condition at periodic intervals during storage

\begin{tabular}{|c|c|c|c|c|c|c|c|c|c|c|c|c|c|c|c|c|}
\hline $\begin{array}{l}\text { 0 } \\
\text { DAS }\end{array}$ & $\begin{array}{c}15 \\
\text { DAS }\end{array}$ & Cold & $\begin{array}{c}\text { Ambi } \\
\text { ent }\end{array}$ & $\begin{array}{c}\text { Mea } \\
\text { n A }\end{array}$ & 30 DAS & Cold & $\begin{array}{l}\text { Amb } \\
\text { ient }\end{array}$ & $\begin{array}{c}\text { Mean } \\
\text { A }\end{array}$ & 45 DAS & Cold & $\begin{array}{c}\text { Amb } \\
\text { ient }\end{array}$ & $\begin{array}{c}\text { Mea } \\
\text { n A }\end{array}$ & 60 DAS & Cold & $\begin{array}{c}\text { Ambi } \\
\text { ent }\end{array}$ & $\begin{array}{c}\text { Mea } \\
\text { n A }\end{array}$ \\
\hline \multirow{11}{*}{$\begin{array}{l}\text { A }(1- \\
6) x \\
B(1- \\
2)= \\
10.04\end{array}$} & $\mathbf{T}_{1}$ & 11.20 & 11.57 & 11.39 & $\mathbf{T}_{1}$ & 11.57 & 13.69 & 12.63 & $\mathbf{T}_{1}$ & 12.04 & 15.59 & 13.82 & $\mathbf{T}_{1}$ & 13.69 & 18.89 & 16.29 \\
\hline & $\mathbf{T}_{2}$ & 10.07 & 11.57 & 10.82 & $\mathbf{T}_{2}$ & 11.24 & 13.09 & 12.17 & $\mathbf{T}_{2}$ & 11.57 & 13.69 & 12.63 & $\mathbf{T}_{2}$ & 13.69 & 17.77 & 15.73 \\
\hline & $\mathbf{T}_{\mathbf{3}}$ & 11.20 & 11.57 & 11.39 & $\mathbf{T}_{\mathbf{3}}$ & 11.57 & 13.69 & 12.63 & $\mathbf{T}_{3}$ & 12.04 & 15.59 & 13.82 & $\mathbf{T}_{\mathbf{3}}$ & 13.69 & 23.33 & 18.51 \\
\hline & $\mathbf{T}_{4}$ & 10.07 & 11.24 & 10.65 & $\mathbf{T}_{4}$ & 11.24 & 13.09 & 12.17 & $\mathbf{T}_{4}$ & 11.57 & 13.69 & 12.63 & $\mathbf{T}_{4}$ & 13.09 & 15.59 & 14.34 \\
\hline & $\mathbf{T}_{5}$ & 10.07 & 11.24 & 10.65 & $\mathbf{T}_{5}$ & 11.20 & 12.04 & 11.62 & $\mathbf{T}_{5}$ & 11.57 & 13.69 & 12.63 & $\mathbf{T}_{5}$ & 13.09 & 13.69 & 13.39 \\
\hline & $\mathbf{T}_{6}$ & 11.20 & 11.57 & 11.39 & $\mathbf{T}_{6}$ & 11.57 & 13.09 & 12.33 & $\mathbf{T}_{6}$ & 11.57 & 15.59 & 13.58 & $\mathbf{T}_{6}$ & 13.69 & 18.89 & 16.29 \\
\hline & $\begin{array}{c}\text { Mean } \\
\text { B }\end{array}$ & 10.64 & 11.46 & & Mean B & 11.40 & 13.12 & & Mean B & 11.73 & 14.64 & & Mean B & 13.49 & 18.03 & \\
\hline & $\begin{array}{c}\text { Facto } \\
\text { rs }\end{array}$ & C.D. & $\operatorname{SE}(d)$ & $\begin{array}{l}\text { SE } \\
(\mathbf{m})\end{array}$ & Factors & C.D. & $\begin{array}{l}\text { SE } \\
\text { (d) }\end{array}$ & SE(m) & Factors & C.D. & $\begin{array}{l}\text { SE } \\
\text { (d) }\end{array}$ & $\begin{array}{l}\text { SE } \\
(\mathbf{m})\end{array}$ & Factors & C.D. & $\operatorname{SE}(d)$ & $\begin{array}{c}\text { SE( } \\
\text { m) }\end{array}$ \\
\hline & $\begin{array}{c}\text { Facto } \\
\text { r(A) }\end{array}$ & 0.106 & 0.051 & 0.036 & $\begin{array}{c}\text { Factor } \\
\text { (A) }\end{array}$ & 0.084 & 0.041 & 0.029 & $\begin{array}{c}\text { Factor } \\
\text { (A) }\end{array}$ & 0.102 & 0.049 & 0.035 & $\begin{array}{l}\text { Factor } \\
\text { (A) }\end{array}$ & 0.093 & 0.045 & 0.032 \\
\hline & $\begin{array}{c}\text { Facto } \\
\mathbf{r}(\mathbf{B})\end{array}$ & 0.061 & 0.029 & 0.021 & $\begin{array}{c}\text { Factor } \\
\text { (B) }\end{array}$ & 0.049 & 0.023 & 0.017 & $\begin{array}{c}\text { Factor } \\
\text { (B) }\end{array}$ & 0.059 & 0.028 & 0.020 & $\begin{array}{l}\text { Factor } \\
\text { (B) }\end{array}$ & 0.054 & 0.026 & 0.018 \\
\hline & $\begin{array}{c}\text { Facto } \\
\text { r } \\
\text { (A X } \\
\text { B ) }\end{array}$ & 0.150 & 0.072 & 0.051 & $\begin{array}{c}\text { Factor } \\
(\text { A X B ) }\end{array}$ & 0.119 & 0.057 & 0.041 & $\begin{array}{c}\text { Factor } \\
(\text { A X B ) }\end{array}$ & 0.145 & 0.070 & 0.049 & $\begin{array}{c}\text { Factor } \\
\text { (A X B) }\end{array}$ & 0.132 & 0.063 & 0.045 \\
\hline
\end{tabular}

( $\mathrm{T}_{1}$ - HDPE, $\mathrm{T}_{2}$-Styrofoam, $\mathrm{T}_{3}$ - Brown paper, $\mathrm{T}_{4^{-}}$Plastic punnet box, $\mathrm{T}_{5^{-}}$Styrofoam + $\mathrm{LDPE}, \mathrm{T}_{6^{-}}$LDPE $+\mathrm{HDPE}$ ) 
Table.4 Titratable acidity (\%) of pomegranate leathers packaged in different packaging treatments in cold and ambient storage condition at periodic intervals during storage

\begin{tabular}{|c|c|c|c|c|c|c|c|c|c|c|c|c|c|c|c|c|}
\hline $\begin{array}{c}\text { 0 } \\
\text { DAS }\end{array}$ & $\begin{array}{c}15 \\
\text { DAS }\end{array}$ & Cold & $\begin{array}{c}\text { Ambi } \\
\text { ent }\end{array}$ & $\begin{array}{c}\text { Mea } \\
\text { n A }\end{array}$ & 30 DAS & Cold & $\begin{array}{c}\text { Am } \\
\text { bien } \\
\mathbf{t}\end{array}$ & $\begin{array}{c}\text { Mea } \\
\text { n A }\end{array}$ & 45 DAS & $\begin{array}{l}\text { Col } \\
\text { d }\end{array}$ & $\begin{array}{c}\text { Amb } \\
\text { ient }\end{array}$ & $\begin{array}{c}\text { Mea } \\
\text { n A }\end{array}$ & 60 DAS & $\begin{array}{l}\text { Col } \\
\text { d }\end{array}$ & $\begin{array}{c}\text { Amb } \\
\text { ient }\end{array}$ & $\begin{array}{c}\text { Mea } \\
\text { n A }\end{array}$ \\
\hline \multirow{11}{*}{$\begin{array}{c}\text { A (1- } \\
\text { 6) } x \\
\text { B }(1- \\
2)= \\
0.06\end{array}$} & $\mathbf{T}_{1}$ & 0.12 & 0.19 & 0.16 & $\mathbf{T}_{1}$ & 0.19 & 0.25 & 0.22 & $\mathbf{T}_{1}$ & 0.23 & 0.32 & 0.28 & $\mathbf{T}_{1}$ & 0.30 & 0.42 & 0.36 \\
\hline & $\mathbf{T}_{2}$ & 0.12 & 0.12 & 0.12 & $\mathbf{T}_{2}$ & 0.19 & 0.23 & 0.21 & $\mathbf{T}_{2}$ & 0.19 & 0.30 & 0.24 & $\mathbf{T}_{2}$ & 0.23 & 0.36 & 0.30 \\
\hline & $\mathbf{T}_{3}$ & 0.12 & 0.19 & 0.16 & $\mathbf{T}_{3}$ & 0.21 & 0.30 & 0.25 & $\mathbf{T}_{\mathbf{3}}$ & 0.25 & 0.32 & 0.29 & $\mathbf{T}_{3}$ & 0.30 & 0.42 & 0.36 \\
\hline & $\mathbf{T}_{4}$ & 0.06 & 0.06 & 0.06 & $\mathbf{T}_{4}$ & 0.19 & 0.19 & 0.19 & $\mathbf{T}_{4}$ & 0.19 & 0.25 & 0.22 & $\mathbf{T}_{4}$ & 0.23 & 0.30 & 0.26 \\
\hline & $\mathbf{T}_{5}$ & 0.06 & 0.12 & 0.09 & $\mathbf{T}_{5}$ & 0.12 & 0.23 & 0.18 & $\mathbf{T}_{5}$ & 0.19 & 0.30 & 0.24 & $\mathbf{T}_{5}$ & 0.19 & 0.32 & 0.26 \\
\hline & $\mathbf{T}_{6}$ & 0.12 & 0.19 & 0.16 & $\mathbf{T}_{6}$ & 0.19 & 0.25 & 0.22 & $\mathbf{T}_{6}$ & 0.23 & 0.32 & 0.28 & $\mathbf{T}_{6}$ & 0.23 & 0.42 & 0.33 \\
\hline & $\begin{array}{c}\text { Mean } \\
\text { B }\end{array}$ & 0.10 & 0.15 & & Mean B & 0.18 & 0.24 & & Mean B & 0.21 & 0.30 & & Mean B & 0.25 & 0.37 & \\
\hline & $\begin{array}{c}\text { Factor } \\
\mathrm{s} \\
\end{array}$ & C.D. & SE(d) & $\begin{array}{l}\text { SE } \\
(\mathbf{m})\end{array}$ & Factors & C.D. & $\begin{array}{l}\text { SE } \\
\text { (d) }\end{array}$ & $\begin{array}{l}\text { SE } \\
(\mathbf{m})\end{array}$ & Factors & C.D. & $\begin{array}{l}\text { SE } \\
\text { (d) }\end{array}$ & $\begin{array}{l}\text { SE } \\
(\mathbf{m})\end{array}$ & Factors & $\begin{array}{c}\text { C.D } \\
.\end{array}$ & $\begin{array}{l}\text { SE } \\
\text { (d) }\end{array}$ & $\begin{array}{l}\text { SE } \\
(\mathbf{m})\end{array}$ \\
\hline & $\begin{array}{l}\text { Factor } \\
\text { (A) }\end{array}$ & 0.023 & 0.011 & 0.008 & $\begin{array}{l}\text { Factor } \\
\text { (A) }\end{array}$ & 0.025 & $\begin{array}{c}0.01 \\
2\end{array}$ & $\begin{array}{c}0.00 \\
9\end{array}$ & $\begin{array}{l}\text { Factor } \\
\text { (A) }\end{array}$ & $\begin{array}{c}0.02 \\
6\end{array}$ & 0.013 & $\begin{array}{c}0.00 \\
9\end{array}$ & $\begin{array}{c}\text { Factor } \\
\text { (A) }\end{array}$ & $\begin{array}{c}0.05 \\
6\end{array}$ & $\begin{array}{c}0.02 \\
7\end{array}$ & $\begin{array}{c}0.01 \\
9\end{array}$ \\
\hline & $\begin{array}{l}\text { Factor } \\
\text { (B) }\end{array}$ & 0.013 & 0.006 & 0.004 & $\begin{array}{c}\text { Factor } \\
\text { (B) }\end{array}$ & 0.014 & $\begin{array}{c}0.00 \\
7\end{array}$ & $\begin{array}{c}0.00 \\
5\end{array}$ & $\begin{array}{l}\text { Factor } \\
\text { (B) }\end{array}$ & $\begin{array}{c}0.01 \\
5\end{array}$ & 0.007 & $\begin{array}{c}0.00 \\
5\end{array}$ & $\begin{array}{c}\text { Factor } \\
\text { (B) }\end{array}$ & $\begin{array}{c}0.03 \\
3\end{array}$ & $\begin{array}{c}0.01 \\
6\end{array}$ & $\begin{array}{c}0.01 \\
1\end{array}$ \\
\hline & $\begin{array}{c}\text { Factor } \\
\text { (A X } \\
\text { B) }\end{array}$ & 0.032 & 0.015 & 0.011 & $\begin{array}{c}\text { Factor } \\
\text { (A X B) }\end{array}$ & 0.035 & $\begin{array}{c}0.01 \\
7\end{array}$ & $\begin{array}{c}0.01 \\
2\end{array}$ & $\begin{array}{l}\text { Factor } \\
(\text { A X B) }\end{array}$ & N/A & 0.018 & $\begin{array}{c}0.01 \\
3\end{array}$ & $\begin{array}{c}\text { Factor( } \\
\text { A X B) }\end{array}$ & N/A & $\begin{array}{c}0.03 \\
8\end{array}$ & $\begin{array}{c}0.02 \\
7\end{array}$ \\
\hline
\end{tabular}

$\left(\mathrm{T}_{1}\right.$ - HDPE, $\mathrm{T}_{2}$-Styrofoam, $\mathrm{T}_{3^{-}}$Brown paper, $\mathrm{T}_{4^{-}}$Plastic punnet box, $\mathrm{T}_{5^{-}}$Styrofoam $+\mathrm{LDPE}, \mathrm{T}_{6^{-}}$LDPE $\left.+\mathrm{HDPE}\right)$ 
Table.5 Antioxidant content (percent inhibition of DPPH) of pomegranate leathers packaged in different packaging treatments in cold and ambient storage condition at periodic intervals during storage

\begin{tabular}{|c|c|c|c|c|c|c|c|c|c|c|c|c|c|c|c|c|}
\hline $\begin{array}{c}\text { 0 } \\
\text { DAS }\end{array}$ & 15 DAS & Cold & $\begin{array}{c}\text { Ambi } \\
\text { ent }\end{array}$ & $\begin{array}{c}\text { Mea } \\
\text { n A }\end{array}$ & 30 DAS & Cold & $\begin{array}{c}\text { Ambie } \\
\text { nt }\end{array}$ & $\begin{array}{c}\text { Mea } \\
\text { n A }\end{array}$ & 45 DAS & Cold & $\begin{array}{c}\text { Ambi } \\
\text { ent }\end{array}$ & $\begin{array}{c}\text { Mean } \\
\text { A }\end{array}$ & 60 DAS & Cold & $\begin{array}{c}\text { Ambi } \\
\text { ent }\end{array}$ & $\begin{array}{c}\text { Mea } \\
\text { n A }\end{array}$ \\
\hline \multirow{11}{*}{$\begin{array}{c}\text { A }(1- \\
6) \times \\
\text { B }(1- \\
2)=79 \\
.45\end{array}$} & $\mathbf{T}_{1}$ & 78.91 & 78.14 & 78.52 & $\mathbf{T}_{1}$ & 78.14 & 65.47 & 71.80 & $\mathbf{T}_{1}$ & $\begin{array}{c}74.2 \\
3\end{array}$ & 50.08 & 62.16 & $\mathbf{T}_{1}$ & $\begin{array}{c}66.8 \\
3\end{array}$ & 18.42 & $\begin{array}{c}42.6 \\
3\end{array}$ \\
\hline & $\mathbf{T}_{2}$ & 79.33 & 78.14 & 78.73 & $\mathbf{T}_{2}$ & 78.57 & 66.83 & 72.70 & $\mathbf{T}_{2}$ & $\begin{array}{c}74.2 \\
3\end{array}$ & 55.95 & 65.09 & $\mathbf{T}_{2}$ & $\begin{array}{c}69.3 \\
8\end{array}$ & 29.93 & $\begin{array}{c}49.6 \\
6\end{array}$ \\
\hline & $\mathbf{T}_{3}$ & 78.57 & 78.14 & 78.35 & $\mathbf{T}_{\mathbf{3}}$ & 78.14 & 65.47 & 71.80 & $\mathbf{T}_{\mathbf{3}}$ & $\begin{array}{c}69.3 \\
9\end{array}$ & 48.21 & 58.80 & $\mathbf{T}_{\mathbf{3}}$ & $\begin{array}{c}66.8 \\
3\end{array}$ & 16.67 & $\begin{array}{c}41.7 \\
5\end{array}$ \\
\hline & $\mathbf{T}_{4}$ & 79.33 & 78.57 & 78.95 & $\mathbf{T}_{4}$ & 78.57 & 74.23 & 76.40 & $\mathbf{T}_{4}$ & $\begin{array}{c}77.8 \\
9\end{array}$ & 55.95 & 66.92 & $\mathbf{T}_{4}$ & $\begin{array}{c}74.2 \\
3\end{array}$ & 31.97 & $\begin{array}{c}53.1 \\
0\end{array}$ \\
\hline & $\mathbf{T}_{5}$ & 79.33 & 78.57 & 78.95 & $\mathbf{T}_{5}$ & 79.33 & 69.39 & 74.36 & $\mathbf{T}_{5}$ & $\begin{array}{c}77.8 \\
9\end{array}$ & 55.95 & 66.92 & $\mathbf{T}_{5}$ & $\begin{array}{c}74.2 \\
3\end{array}$ & 29.93 & $\begin{array}{c}52.0 \\
8\end{array}$ \\
\hline & $\mathbf{T}_{6}$ & 78.91 & 78.14 & 78.52 & $\mathbf{T}_{6}$ & 78.57 & 66.83 & 72.70 & $\mathbf{T}_{6}$ & $\begin{array}{c}74.2 \\
3\end{array}$ & 50.08 & 62.16 & $\mathbf{T}_{6}$ & $\begin{array}{c}66.8 \\
3\end{array}$ & 29.93 & $\begin{array}{c}48.3 \\
8\end{array}$ \\
\hline & Mean B & 79.06 & 78.28 & & $\begin{array}{c}\text { Mean } \\
\text { B }\end{array}$ & 78.55 & 68.04 & & Mean B & $\begin{array}{c}74.6 \\
4\end{array}$ & 52.70 & & Mean B & $\begin{array}{c}69.7 \\
2\end{array}$ & 26.14 & \\
\hline & Factors & C.D. & $\operatorname{SE}(d)$ & $\begin{array}{l}\text { SE( } \\
\text { m) }\end{array}$ & Factors & C.D. & $\operatorname{SE}(d)$ & $\begin{array}{l}\text { SE } \\
(\mathbf{m})\end{array}$ & Factors & C.D. & $\begin{array}{l}\text { SE } \\
\text { (d) }\end{array}$ & $\mathrm{SE}(\mathrm{m})$ & Factors & C.D. & $\begin{array}{l}\text { SE } \\
\text { (d) }\end{array}$ & $\begin{array}{l}\text { SE } \\
\text { (m) }\end{array}$ \\
\hline & $\begin{array}{c}\text { Factor } \\
\text { (A) }\end{array}$ & 0.120 & 0.058 & 0.041 & $\begin{array}{c}\text { Factor } \\
\text { (A) }\end{array}$ & 2.091 & 1.007 & 0.712 & $\begin{array}{c}\text { Factor } \\
\text { (A) }\end{array}$ & $\begin{array}{c}0.24 \\
9\end{array}$ & 0.120 & 0.085 & $\begin{array}{c}\text { Factor } \\
\text { (A) }\end{array}$ & $\begin{array}{c}0.76 \\
9\end{array}$ & 0.370 & $\begin{array}{c}0.26 \\
2\end{array}$ \\
\hline & $\begin{array}{c}\text { Factor } \\
\text { (B) }\end{array}$ & 0.069 & 0.033 & 0.024 & $\begin{array}{l}\text { Factor } \\
\text { (B) }\end{array}$ & 1.207 & 0.581 & 0.411 & $\begin{array}{c}\text { Factor } \\
\text { (B) }\end{array}$ & $\begin{array}{c}0.14 \\
4\end{array}$ & 0.069 & 0.049 & $\begin{array}{c}\text { Factor } \\
\text { (B) }\end{array}$ & $\begin{array}{c}0.44 \\
4\end{array}$ & 0.214 & $\begin{array}{c}0.15 \\
1\end{array}$ \\
\hline & $\begin{array}{c}\text { Factor } \\
\text { (A X B) }\end{array}$ & 0.170 & 0.082 & 0.058 & $\begin{array}{c}\text { Factor } \\
\text { (A X B) }\end{array}$ & 2.957 & 1.424 & 1.007 & $\begin{array}{c}\text { Factor } \\
\text { (A X B) }\end{array}$ & $\begin{array}{c}0.35 \\
3\end{array}$ & 0.170 & 0.120 & $\begin{array}{l}\text { Factor( } \\
\text { A X B) }\end{array}$ & $\begin{array}{c}1.08 \\
7\end{array}$ & 0.524 & $\begin{array}{c}0.37 \\
0\end{array}$ \\
\hline
\end{tabular}

$\left(\mathrm{T}_{1}\right.$ - HDPE, $\mathrm{T}_{2}$-Styrofoam, $\mathrm{T}_{3}$ - Brown paper, $\mathrm{T}_{4}$ - Plastic punnet box, $\mathrm{T}_{5}$ - Styrofoam + LDPE, $\mathrm{T}_{6}$ - LDPE + HDPE) 
Table.6 Total phenol content (mgGAE/g) of pomegranate leathers packaged in different packaging treatments in cold and ambient storage condition at periodic intervals during storage

\begin{tabular}{|c|c|c|c|c|c|c|c|c|c|c|c|c|c|c|c|c|}
\hline 0 DAS & 15 DAS & Cold & $\begin{array}{c}\text { Ambi } \\
\text { ent }\end{array}$ & $\begin{array}{c}\text { Mea } \\
\text { n A }\end{array}$ & $\begin{array}{c}30 \\
\text { DAS }\end{array}$ & $\begin{array}{c}\text { Col } \\
\text { d }\end{array}$ & $\begin{array}{c}\text { Am } \\
\text { bien } \\
\mathbf{t}\end{array}$ & $\begin{array}{c}\text { Mea } \\
\text { n A }\end{array}$ & $\begin{array}{c}45 \\
\text { DAS }\end{array}$ & $\begin{array}{c}\text { Col } \\
\text { d }\end{array}$ & $\begin{array}{c}\text { Am } \\
\text { bien } \\
\mathbf{t}\end{array}$ & $\begin{array}{c}\text { Mea } \\
\text { n A }\end{array}$ & 60 DAS & Cold & $\begin{array}{r}\text { Amb } \\
\text { ient }\end{array}$ & $\begin{array}{c}\text { Mean } \\
\text { A }\end{array}$ \\
\hline \multirow{11}{*}{$\begin{array}{c}\text { A }(1-6) \\
\times \text { B }(1- \\
2)= \\
23.04\end{array}$} & $\mathbf{T}_{1}$ & 20.35 & 14.66 & $\begin{array}{c}17.5 \\
1\end{array}$ & $\mathbf{T}_{1}$ & $\begin{array}{c}15.9 \\
6\end{array}$ & $\begin{array}{c}10.7 \\
4\end{array}$ & $\begin{array}{c}13.3 \\
5\end{array}$ & $\mathbf{T}_{1}$ & $\begin{array}{c}15.2 \\
5\end{array}$ & 8.25 & $\begin{array}{c}11.7 \\
5\end{array}$ & $\mathbf{T}_{1}$ & 13.11 & 4.65 & 8.88 \\
\hline & $\mathbf{T}_{2}$ & 21.07 & 21.10 & $\begin{array}{c}21.0 \\
9\end{array}$ & $\mathbf{T}_{2}$ & $\begin{array}{c}16.1 \\
4\end{array}$ & $\begin{array}{c}12.8 \\
9\end{array}$ & $\begin{array}{c}14.5 \\
2\end{array}$ & $\mathbf{T}_{2}$ & $\begin{array}{c}15.4 \\
9 \\
\end{array}$ & 9.43 & $\begin{array}{c}12.4 \\
6 \\
\end{array}$ & $\mathbf{T}_{2}$ & 13.44 & 6.41 & 9.93 \\
\hline & $\mathbf{T}_{\mathbf{3}}$ & 20.35 & 14.66 & $\begin{array}{c}17.5 \\
1\end{array}$ & $\mathbf{T}_{\mathbf{3}}$ & $\begin{array}{c}15.9 \\
6\end{array}$ & $\begin{array}{c}10.5 \\
5\end{array}$ & $\begin{array}{c}13.2 \\
6\end{array}$ & $\mathbf{T}_{\mathbf{3}}$ & $\begin{array}{c}15.2 \\
5\end{array}$ & 8.02 & $\begin{array}{c}11.6 \\
4\end{array}$ & $\mathbf{T}_{\mathbf{3}}$ & 12.29 & 3.78 & 8.04 \\
\hline & $\mathbf{T}_{4}$ & 21.07 & 20.35 & $\begin{array}{c}20.7 \\
1\end{array}$ & $\mathbf{T}_{4}$ & $\begin{array}{c}17.5 \\
7\end{array}$ & $\begin{array}{c}15.7 \\
7\end{array}$ & $\begin{array}{c}16.6 \\
7\end{array}$ & $\mathbf{T}_{4}$ & $\begin{array}{c}15.6 \\
9\end{array}$ & $\begin{array}{c}10.1 \\
3\end{array}$ & $\begin{array}{c}12.9 \\
1\end{array}$ & $\mathbf{T}_{4}$ & 13.61 & 7.13 & 10.37 \\
\hline & $\mathbf{T}_{5}$ & 22.66 & 20.35 & $\begin{array}{c}21.5 \\
1\end{array}$ & $\mathbf{T}_{5}$ & $\begin{array}{c}18.7 \\
7\end{array}$ & $\begin{array}{c}13.1 \\
1\end{array}$ & $\begin{array}{c}15.9 \\
4\end{array}$ & $\mathbf{T}_{5}$ & $\begin{array}{c}16.0 \\
5\end{array}$ & 9.98 & $\begin{array}{c}13.0 \\
2\end{array}$ & $\mathbf{T}_{5}$ & 14.66 & 7.03 & 10.85 \\
\hline & $\mathbf{T}_{6}$ & 21.07 & 16.14 & $\begin{array}{c}18.6 \\
1\end{array}$ & $\mathbf{T}_{6}$ & $\begin{array}{c}16.0 \\
5\end{array}$ & $\begin{array}{c}12.0 \\
5\end{array}$ & $\begin{array}{c}14.0 \\
5\end{array}$ & $\mathbf{T}_{6}$ & $\begin{array}{c}15.4 \\
9\end{array}$ & 9.01 & $\begin{array}{c}12.2 \\
5\end{array}$ & $\mathbf{T}_{6}$ & 13.11 & 5.64 & 9.38 \\
\hline & Mean B & 21.10 & 17.88 & & $\begin{array}{c}\text { Mean } \\
\text { B }\end{array}$ & $\begin{array}{c}16.7 \\
4\end{array}$ & $\begin{array}{c}12.5 \\
2\end{array}$ & & $\begin{array}{c}\text { Mean } \\
\text { B }\end{array}$ & $\begin{array}{c}15.5 \\
4\end{array}$ & 9.14 & & Mean B & 13.37 & 5.77 & \\
\hline & Factors & C.D. & SE(d) & $\begin{array}{l}\text { SE } \\
(\mathbf{m})\end{array}$ & $\begin{array}{c}\text { Factor } \\
\mathbf{S}\end{array}$ & C.D. & $\begin{array}{l}\text { SE } \\
\text { (d) }\end{array}$ & $\begin{array}{l}\text { SE } \\
(\mathbf{m})\end{array}$ & $\begin{array}{c}\text { Factor } \\
\mathbf{S}\end{array}$ & C.D. & $\begin{array}{l}\text { SE( } \\
\text { d) }\end{array}$ & $\begin{array}{l}\text { SE } \\
(\mathbf{m})\end{array}$ & Factors & C.D. & $\begin{array}{l}\text { SE } \\
\text { (d) }\end{array}$ & $\begin{array}{l}\text { SE } \\
(\mathbf{m})\end{array}$ \\
\hline & $\begin{array}{l}\text { Factor } \\
\text { (A) }\end{array}$ & 0.334 & 0.161 & $\begin{array}{c}0.11 \\
4\end{array}$ & $\begin{array}{l}\text { Factor } \\
\text { (A) }\end{array}$ & $\begin{array}{c}0.49 \\
5\end{array}$ & $\begin{array}{c}0.23 \\
8\end{array}$ & $\begin{array}{c}0.16 \\
9\end{array}$ & $\begin{array}{l}\text { Factor } \\
\text { (A) }\end{array}$ & $\begin{array}{c}0.21 \\
1\end{array}$ & $\begin{array}{c}0.10 \\
2\end{array}$ & $\begin{array}{c}0.07 \\
2\end{array}$ & $\begin{array}{l}\text { Factor } \\
\text { (A) }\end{array}$ & 0.467 & $\begin{array}{c}0.22 \\
5\end{array}$ & 0.159 \\
\hline & $\begin{array}{c}\text { Factor } \\
\text { (B) }\end{array}$ & 0.193 & 0.093 & $\begin{array}{c}0.06 \\
6\end{array}$ & $\begin{array}{c}\text { Factor } \\
\text { (B) }\end{array}$ & $\begin{array}{c}0.28 \\
6 \\
\end{array}$ & $\begin{array}{c}0.13 \\
8\end{array}$ & $\begin{array}{c}0.09 \\
7\end{array}$ & $\begin{array}{c}\text { Factor } \\
\text { (B) }\end{array}$ & $\begin{array}{c}0.12 \\
2\end{array}$ & $\begin{array}{c}0.05 \\
9\end{array}$ & $\begin{array}{c}0.04 \\
1\end{array}$ & $\begin{array}{c}\text { Factor } \\
\text { (B) }\end{array}$ & 0.270 & $\begin{array}{c}0.13 \\
0\end{array}$ & 0.092 \\
\hline & $\begin{array}{c}\text { Factor } \\
\text { (A X B) }\end{array}$ & 0.473 & 0.228 & $\begin{array}{c}0.16 \\
1\end{array}$ & $\begin{array}{l}\text { Factor } \\
\text { (A X } \\
\text { B) }\end{array}$ & $\begin{array}{c}0.70 \\
0\end{array}$ & $\begin{array}{c}0.33 \\
7\end{array}$ & $\begin{array}{c}0.23 \\
8\end{array}$ & $\begin{array}{c}\text { Factor } \\
(\text { A X } \\
\text { B) }\end{array}$ & $\begin{array}{c}0.29 \\
8\end{array}$ & $\begin{array}{c}0.14 \\
4\end{array}$ & $\begin{array}{c}0.10 \\
2\end{array}$ & $\begin{array}{c}\text { Factor(A } \\
\text { X B) }\end{array}$ & 0.660 & $\begin{array}{c}0.31 \\
8\end{array}$ & 0.225 \\
\hline
\end{tabular}

( $\mathrm{T}_{1}$ - HDPE, $\mathrm{T}_{2}$-Styrofoam, $\mathrm{T}_{3}$ - Brown paper, $\mathrm{T}_{4}$ - Plastic punnet box, $\mathrm{T}_{5}-$ Styrofoam + LDPE, $\mathrm{T}_{6}$ - LDPE + HDPE) 
By the end of the study (for ambient condition) pomegranate leather packed with packaging material $\mathrm{T}_{4}$ (Plastic punnet box) was able to withhold the maximum antioxidant (percentage inhibition of DPPH) level of 31.97 which was followed by pomegranate leather packed with treatment $\mathrm{T}_{2}$ (Styrofoam), $\mathrm{T}_{5}$ (Styrofoam + LDPE) and $\mathrm{T}_{6}$ (LDPE + HDPE) depicting values of 29.93 respectively.

The total phenolic content (Table 6) as like the antioxidant levels decreased with each interval of storage analysis. For the pomegranate leathers with different packaging treatments at refrigerated condition the total phenolic content at 0 days of storage were at its peak which gradually decreased. The total phenolic content (refrigerated condition) at 15 days of storage for all the treatments where above $20 \mathrm{mg} \mathrm{GAE} / \mathrm{g}$, with further decreased at 30, 45, 60 days of storage. At the end of experiment it was seen that among the pomegranate leathers packed with different treatments (refrigerated condition) pomegranate leather packed with $\mathrm{T}_{5}$ (Styrofoam + LDPE), showed the highest values which was followed by pomegranate leathers packed with $\mathrm{T}_{4}$ (Plastic punnet box), $\mathrm{T}_{2}$ (Styrofoam), $\mathrm{T}_{6}$ (LDPE $\left.+\mathrm{HDPE}\right)$ and $\mathrm{T}_{1}$ (HDPE) and lastly by pomegranate leather packed in $\mathrm{T}_{3}$ (Brown paper). A similar kind of observation with decreased values of total phenolic content was seen for pomegranate leathers packed in different packaging materials at ambient storage condition. However the decrease in the total phenolic content was much more expedite here as compared to pomegranate leather stored in refrigerated condition. For the ambient situation (at 60 days after storage) pomegranate leather packed in $\mathrm{T}_{4}$ (Plastic punnet box) maintained the highest phenolic concentration at $7.13 \mathrm{mg}$ GAE/gm followed by pomegranate leather packed in $T_{5}$ (Styrofoam + LDPE) where, the phenolic concentration was at $7.03 \mathrm{mg}$ GAE/gm and pomegranate leather packed with packaging material $\mathrm{T}_{3}$ (Brown paper) depicted the lowest values of $3.77 \mathrm{mg} \mathrm{GAE} / \mathrm{gm}$.

Fungal colony development for pomegranate leather (Fig. 1 and 2) stored at refrigerated condition, was nil at 15 days interval, however at 60 days of storage pomegranate leather packed in $\mathrm{T}_{1}$ (HDPE), $\mathrm{T}_{3}$ (Brown paper) and $\mathrm{T}_{6}$ reported one fungal colony development and no fungal growth was seen for pomegranate leathers packed in $T_{2}$ (Styrofoam), $\mathrm{T}_{4}$ (Plastic punnet box) and $\mathrm{T}_{5}$ (Styrofoam + LDPE). For ambient storage condition, infestation at 15 days interval was seen with 1 fungal colony development in pomegranate leathers packed with $\mathrm{T}_{1}$ (HDPE) and $\mathrm{T}_{6}$ (LDPE + HDPE). Pomegranate leathers packed with $\mathrm{T}_{3}$ (Brown paper) was reported with 2 fungal colony and no growth was seen for pomegranate leathers packed with $\mathrm{T}_{2}$ (Styrofoam), $\mathrm{T}_{4}$ (Plastic punnet box) and $\mathrm{T}_{5}$ (Styrofoam + LDPE). However the infestation for ambient storage at 60 days interval for different pomegranate leathers was seen to certain extends. Pomegranate leathers packed with $\mathrm{T}_{3}$ (Brown paper) was reported with the highest fungal colonies (6 $\mathrm{cfu} / \mathrm{g})$. Pomegranate leathers packed with $\mathrm{T}_{6}$ (LDPE + HDPE) was seen with 4 fungal colonies and 2 fungal colonies were observed for each of the pomegranate leathers packed with $\mathrm{T}_{1}$ (HDPE), $\mathrm{T}_{2}$ (Styrofoam), $\mathrm{T}_{4}$ (Plastic punnet box) and $\mathrm{T}_{5}$ (Styrofoam $\left.{ }^{+} \mathrm{LDPE}\right)$.

In the present study, after preparation of pomegranate leather they were packed into different types of packaging treatments for storage. Similar types of studies where variable packaging material were used for packaging of fruit leather were conducted by Fulchand et al., (2015) and Kumar \& Sukhla, (2018). After packaging, storage was done at refrigerated and ambient conditions and analysis was carried upon at different storage 
intervals. From the results it was seen that the TSS increased both in refrigerated and ambient for all the pomegranate leathers packed with different packaging treatments. Similar results were observed in guava leather (Basha, 2018) and for fig leathers (Kotlawar, 2008). Likewise the titratable acidity, total sugar and reducing sugar increased for the pomegranate leathers stored in ambient and refrigerated storage, but the rate of increase was observed more for the packed pomegranate leathers stored in ambient condition as compared to refrigerated ones. The results were at par to the observation of Basha, 2018 with respect to guava fruit leather. The antioxidant and phenol decreased during the storage period of analysis. Findings were in accordance with previous work of Das and Dhua, (2019) on dried banana inflorescence.

Over all from the study we can conclude that among the storage conditions refrigerated situation was better as compared to the ambient condition for storing of pomegranate leather. Regarding the packaging materials or combinations it was found that packaging material with combination of Styrofoam and LDPE and packaging material plastic punnet box, both were more or less suitable for packaging and storing of dehydrated pomegranate leathers at refrigerated and ambient storage conditions.

\section{References}

Allen, O.N. 1953. Experiments in Soil Bacteriology. Burgess Co., Minneapolis, Minn. pp. 69-70.

Basha, S.J. 2018. Effect of Storage Period on Physio - Chemical Properties of Guava Fruit Leather. Int.J.Curr.Microbiol.App.Sci. $\quad 7(04)$ : 1738-1751.

Brand-Williams, W., Cuvelier, M.E. and Berset, C. 1995. Use of a free radical method to evaluate antioxidant activity. LWT Food Sci Tech., 28: 25-30.

Das, A and Dhua, R.S. 2019. Standardization of drying techniques to develop ready to cook banana inflorescence. Int.J.Curr.Microbiol.App.Sci; $\quad 8(3)$ : 1523-1536.

Fulchand, C.R., Gunvantrao, J.V. and Pralhad, I.M. 2015. Studies on effect of drying temperature and storage time on vitamin-c retention capacity and moisture content of papaya-apple fruit leather. Asian Journal of Dairy and Food Research; 34(4): 319-323.

Irtwange, S.V. 2006. Application of modified atmosphere packaging and related technology in postharvest handling of fresh fruits and vegetables. Agric. Eng. Int., 4:1-12.

Kordylas, J.M. 1990. Processing and Preservation of Tropical and Subtropical Foods.Macmillan Publishers Ltd. London.

Kotlawar, N.G. 2008. Investigations on preparation and shelf life of fig leather. M.Sc. Thesis., M.P.K.V., Rahuri.

Kumar, D and Shukla, R.N. 2018. Evaluation of qualitative attributes of papaya leather. International Journal of Agricultural Engineering; 11(1): 84-89.

Rangana, S. 2003. Handbook of Analysis and Quality Control for Fruit and Vegetables Products $2^{\text {nd }}$ ed., Tata McGraw Hill. pp., 12-16.

Sheoran, O.P., Tonk, D.S., Kaushik, L.S., Hasija, R.C. and Pannu, R.S. 1998. Statistical Software Package for Agricultural Research Worker. Recent Advances in information theory, Statistics and Computer Applications by D.S. Hooda and R.C. Hasija, Department of Mathematics Statistics, CCS HAU, Hisar (139-143).

Singleton, V.L., Orthofer, R. and LamuelaRaventos, R.M. 1999. Analysis of total phenols and other oxidation substrates 
and antioxidants by means of FolinCiocalteau reagent. Methods Enzymol., 299: 152-178.

Wills, R.B.H., McGlasson, W.B., Graham, D., Lee, T.H. and Hall, E.G. 1989.
Postharvest-An introduction to the physiology and handling of fruit and vegtables, 3rd edn., New York, U.S.A., Van Nostrand Reinhold p. 46

\section{How to cite this article:}

Kaustubh Das, Mohit Kumar and Ankan Das. 2019. Standardization of Packaging Material and Storage Condition for Pomegranate Leather. Int.J.Curr.Microbiol.App.Sci. 8(08): 2748-2760. doi: https://doi.org/10.20546/ijcmas.2019.808.317 